\title{
Rainfall intermittency and vegetation feedbacks in drylands
}

\author{
M. Baudena ${ }^{1,2}$ and A. Provenzale ${ }^{2}$ \\ ${ }^{1}$ CIMA, Centro di ricerca Interuniversitario in Monitoraggio Ambientale, Via A. Magliotto 8, 17100 Savona, Italy \\ ${ }^{2}$ ISAC-CNR, Istituto di Scienze dell' Atmosfera e del Clima, Corso Fiume 4, 10133 Torino, Italy
}

Received: 2 November 2007 - Published in Hydrol. Earth Syst. Sci. Discuss.: 22 November 2007

Revised: 11 March 2008 - Accepted: 25 March 2008 - Published: 14 April 2008

\begin{abstract}
We discuss a simplifed, conceptual model for the dynamics of the soil-vegetation system in drylands. The model considers the different dynamical processes taking place in vegetated and non-vegetated soil and it distinguishes between the upper soil layer, where rapid evaporation dominates, and the deeper root layer where only plant transpiration takes place. We explore the role of rainfall intermittency and of different plant colonization strategies, and discuss in detail the effect of two different vegetation feedbacks: reduced evaporation due to plant shading and increased infiltration in vegetated areas. The results of the analysis indicate that both temporal rainfall intermittency and the shading/infiltration feedbacks have a beneficial effect on vegetation. However, it turns out that in this model rainfall intermittency and vegetation feedbacks have almost a mutually exclusive role: whenever one of these two components is present, the addition of the other does not further affect vegetation dynamics in a significant way.
\end{abstract}

\section{Introduction}

Understanding the interplay of climate and biosphere is one of the intriguing open issues in Earth System Science. In terrestrial environments, vegetation is affected by atmospheric and soil conditions, and at the same time it influences the atmosphere and the soil, through fluxes of water, energy, and carbon dioxide.

The network of feedbacks between vegetation, climate and soil is, in general, difficult to disentangle, and it is often helpful to study only a portion of the whole system. In such an exercise, simplified, conceptual models can be of much value to elucidate the basic mechanisms at work and identify rele-

Correspondence to: M. Baudena (m.baudena@isac.cnr.it) vant parameters and processes. In this work we follow such a minimalistic approach and discuss a mathematical model for the response of vegetation to the presence or absence of various soil-vegetation feedbacks in arid and semi-arid regions. We assume that the only limiting factor is water and we make the simplifying hypothesis that plant dynamics depends only on soil moisture, similarly to the approach described in the book of Rodriguez-Iturbe and Porporato (2005). Vegetation dynamics is described by an implicit-space approach, as discussed in the works of Levins (1969) and Tilman (1994). In this model, rainfall is the external input, either kept constant or assumed to be stochastic and intermittent in time, and we ignore the feedbacks of vegetation on atmospheric dynamics.

In a previous work (Baudena et al., 2007, BA in the following), we introduced an ecohydrological box model, simpler than the one described here, to estimate the effect of intermittent water availability on vegetation dynamics. The results of that work showed that temporal rainfall intermittency allows for vegetation persistence at low values of annual rainfall volume, where it would go extinct if rainfall were constant. Rainfall intermittency also generates long-term fluctuations in vegetation cover, even in the absence of significant interannual variations in the statistical properties of precipitation.

On the other hand, vegetation modifies the environment where it lives in many different ways, other than those addressed in BA. In arid climates, vegetation diminishes evaporation due to its shadowing effects (Zeng et al., 2006; D'Odorico et al., 2005), and it facilitates infiltration with respect to bare soil surfaces (Rietkerk and van de Koppel, 1997; West, 1990; Walker et al., 1981), a process that has been extensively analyzed with explicit-space models of vegetation patterns in drylands (e.g. von Hardenberg et al., 2001; Rietkerk et al., 2002; Gilad et al., 2004, 2007). Both mechanisms result in a positive feedback that favors vegetation persistence.

The simple approach of BA cannot properly capture the two processes described above: In that model, it is not

Published by Copernicus Publications on behalf of the European Geosciences Union. 
possible to represent the higher infiltration occurring in vegetated soil as compared to bare soil, as soil moisture is spatially averaged over vegetated and non vegetated surfaces. In addition, in BA evaporation was assumed to occur from the whole root layer, whereas in arid and semi-arid regions only the first $5-10 \mathrm{~cm}$ of bare soil dry out, owing to the strong and rapid evaporation, while the underlying soil layer remains wet and functions as water storage (Mahrt and Pan, 1984; Allen et al., 2005; D'Odorico et al., 2005).

To model the infiltration and shading feedbacks, in this work we separately consider the humidity of bare and vegetated soils. Besides, the soil is now divided in two horizontal layers: a thin layer at the surface, where evaporation is intense, and a deeper layer for the underneath root zone. In this way, the model has a total of three soil compartments: the surface layer in either vegetated or non-vegetated soil, and the deeper root layer in vegetated soil. This approach easily allows for a representation of evaporation from the top soil layer only and for a distinction between moisture in vegetated and bare soils.

The rest of this paper is organized as follows. Section 2 provides an introduction to the model adopted here. Section 3 reports the main results of our numerical exploration, and Sect. 4 provides a discussion of our results. Summary and conclusions are reported in Sect. 5.

\section{Model description}

The model adopted here describes the coupled dynamics of vegetation and soil moisture, and it is an extension of the model discussed in BA. Soil moisture dynamics is described following the approach of Laio et al. (2001), and vegetation dynamics is described by an equation analogous to that introduced by Levins (1969) and Tilman (1994).

The soil is divided into top and bottom layers, allowing the representation of evaporation processes taking place only from the upper layer of soil, whereas from the deeper soil layer only transpiration takes place, due to root uptake. Soil moisture is modeled separately for vegetated and bare soil regions, and differential infiltration occurs between bare soil and vegetation. We do not explicitely model soil moisture in the deep layer in bare soil, as we assumed that the water stored there cannot be used by plants and it is effectively lost from the system. The system dynamics is thus modeled by four ordinary differential equations (ODEs) that determine the evolution of four prognostic variables: $s_{e}$, the soil moisture in the top layer of bare (empty) soil, Eq. (1); $s_{u}$ and $s_{d}$, the soil moisture in respectively the top and bottom (root) layers of vegetated sites, Eq. (2)-(3); $b$, the fraction of soil covered by vegetation, Eq. (4):

$$
\begin{aligned}
& n Z_{u} \frac{\mathrm{d} s_{e}}{\mathrm{~d} t}=I\left(s_{e}, f r\right)-E\left(s_{e}\right)-L\left(s_{e}\right) \\
& n Z_{u} \frac{\mathrm{d} s_{u}}{\mathrm{~d} t}=I\left[s_{u}, r+(1-f) r \frac{1-b}{b+\epsilon}\right]-\sigma E\left(s_{u}\right)-L\left(s_{u}\right)
\end{aligned}
$$

$$
\begin{aligned}
n Z_{d} \frac{\mathrm{d} s_{d}}{\mathrm{~d} t}=I_{d}\left[s_{u}, s_{d}, r+\right. & \left.(1-f) r \frac{1-b}{b+\epsilon}\right]+ \\
& +L\left(s_{u}\right)-T\left(s_{d}\right)-L\left(s_{d}\right)
\end{aligned}
$$

$$
\frac{\mathrm{d} b}{\mathrm{~d} t}=g\left(s_{e}, s_{d}\right) b(1-b)-\mu\left(s_{d}\right) b
$$

The relative soil moisture content $\left(0 \leq s_{e}, s_{u}, s_{d} \leq 1\right)$ is averaged over the soil layer (see also e.g. Laio et al., 2001), $n$ is soil porosity, and $Z_{u}=50 \mathrm{~mm}$ and $Z_{d}=250 \mathrm{~mm}$ are the top and bottom layer depths (other reasonable choices provide equivalent results). The quantity $I$ measures the infiltration rate from daily rainfall $r, E$ is the evaporation rate, $T$ is the transpiration rate, and $L$ represents the water loss due to leakage.

Soil moisture dynamics in non-vegetated soil is modeled by Eq. (1). The input is the infiltration from rainfall, $I$, the output is the sum of evaporation, $E$, and leakage, $L$. Rainfall infiltrates in the top soil layer till it saturates, as in Laio et al., 2001:

$$
I\left(s_{e}, \tilde{r}\right)= \begin{cases}\tilde{r} & \text { if } \tilde{r} \Delta t<\left(1-s_{e}\right) n Z_{u} \\ \frac{1-s_{e}}{\Delta t} n Z_{u} & \text { if } \tilde{r} \Delta t \geq\left(1-s_{e}\right) n Z_{u}\end{cases}
$$

where $\Delta t=0.1$ days and $\tilde{r}=f r$. This representation of infiltration will be used also for the surface layer of vegetated soil.

In bare soil, only a fraction $f$ of the total rainfall actually infiltrates, with $0 \leq f \leq 1$. This represents the joint effect of the presence of biophysical crusts that limit infiltration in non-vegetated areas (West, 1990), and of the increased infiltration in vegetated areas due to the presence of roots (e.g. Walker et al., 1981; Tietjen et al., 2007 ${ }^{1}$ ). Losses in bare soil are due to evaporation and leakage. Since the soil is not vegetated, no transpiration occurs. The evaporation rate is zero below a soil moisture threshold corresponding to the hygroscopic point $\left(s_{h}\right)$, then it increases linearly up to the soil field capacity $\left(s_{f c}\right)$, above which it assumes a constant value. The maximum evaporation at field capacity, $E_{f c}=E\left(s_{f c}\right)$, is fixed at about $3.7 \mathrm{~mm}$ per day, in analogy to e.g. Borgogno

\footnotetext{
${ }^{1}$ Tietjen, B., Zehe, E., and Jeltsch, F.: Modelling water availability to plants in drylands under climate change, Water Resour. Res., under revision, 2007.
} 
et al. (2007); D’Odorico et al. (2005). We thus obtain

$$
E= \begin{cases}0 & \text { if } s \leq s_{h} \\ E_{f c} \frac{s-s_{h}}{s_{f c}-s_{h}} & \text { if } s_{h}<s \leq s_{f c} \\ E_{f c} & \text { if } s_{f c}<s \leq 1\end{cases}
$$

Leakage losses occur above the soil field capacity and they are modeled by an exponential growth to the saturated hydraulic conductivity $K_{s}$, reached when the soil is saturated $\left(s_{e}=1\right)$,

$L(s)= \begin{cases}0 & \text { if } s \leq s_{f c} \\ K_{s} \frac{e^{\beta\left(s-s_{f c}\right)}-1}{e^{\beta\left(1-s_{f c}\right)}-1} & \text { if } \quad s_{f c}<s \leq 1\end{cases}$

Leakage losses are represented with Eq. (7) also for surface and deep vegetated soil layers.

Equations (2) and (3) represent the dynamics of relative soil moisture content in the vegetated part. Infiltration occurs in the upper layer up to saturating it, as in bare soil, Eq. (5). The excess water that does not infiltrate in bare soil, $(1-f) r$, is assumed to spread rapidly over the vegetated part of the box, and there it participates in the infiltration process together with the water from local rainfall. Referring to the infiltration as expressed by Eq. (5), the water infiltrating in vegetated soil is therefore

$r^{\prime}=r+(1-f) r \frac{1-b}{b+\epsilon}$,

where $\frac{1-b}{b+\epsilon}$ is a weight used to distribute over the vegetated soil the water which is not infiltrating in bare, crusted areas. To avoid unphysical divergence of infiltration when $b \rightarrow 0$, we introduce a (small) regularizing constant, $\epsilon=0.01$. Evaporation from surface runoff water is assumed to be negligible.

When the top layer saturates, water starts infiltrating into the bottom layer, in analogy to the one-layer approach of e.g. Rodriguez-Iturbe and Porporato, 2005. Infiltration $I_{d}$ into the bottom layer is thus simply modeled as:

$$
\begin{aligned}
& I_{d}\left(s_{u}, s_{d}, r^{\prime}\right)= \\
& \left\{\begin{array}{lc}
0 & \text { if } r^{\prime} \Delta t<\left(1-s_{u}\right) n Z_{u} \\
r^{\prime}-\frac{1-s_{u}}{\Delta t} n Z_{u} & \text { if } 0<r^{\prime} \Delta t-\left(1-s_{u}\right) n Z_{u} \\
1-s_{d} & <\left(1-s_{d}\right) n Z_{d}-L\left(s_{u}\right) \Delta t
\end{array}\right. \\
& \begin{aligned}
\frac{1}{\Delta t} n Z_{d}-L\left(s_{u}\right) & \text { if } r^{\prime} \Delta t-\left(1-s_{u}\right) n Z_{u} \\
& \geq \max \left\{0,\left(1-s_{d}\right) n Z_{d}-L\left(s_{u}\right) \Delta t\right\}
\end{aligned}
\end{aligned}
$$

where $\Delta t=0.1$ days. If the bottom layer gets saturated, water is lost to still deeper layers and cannot be directly used by plant roots. This representation of infiltration is strongly simplified (for a detailed representation, see e.g. Rigby and Porporato, 2006).

Evaporative losses $E$ occur only from the top layer, with the same dependence on soil moisture content expressed by Eq. (6) for bare soil. For consistency with previous works, we call $E_{w}$ the evaporation rate at the value of soil humidity corresponding to the plant wilting point, $s_{w}$ (BA; Borgogno et al., 2007; Laio et al., 2001). With the choices discussed above, we obtain that $E_{w}$ is about $0.4 \mathrm{~mm}$ per day. In BA, $E_{w}$ was lower because evaporation was assumed to occur from the whole root layer.

In vegetated areas, however, plants exert a shadowing effect on the soil, limiting evaporation. This effect is included in the parameter $\sigma$, which multiplies $E$ and can vary from 0 to 1 , representing respectively a total or a null shadowing effect from vegetation cover.

Transpiration contributions from the top layer are implicitly included in the evaporation term, because transpirational losses from the first few centimeters of soil are negligible compared to vegetation shadowing effects (Scanlon and Albertson, 2003; Borgogno et al., 2007; D’Odorico et al., 2005). Thus, we explicitly include a transpiration term $T$ lifting water into the atmosphere from the bottom layer only, as a function of its soil moisture content, $s_{d}$. The transpiration rate is set to zero below the wilting point, $s_{w}$; it grows linearly above $s_{w}$ and assumes a constant value $E_{\max }$ at $s^{*}$, following e.g. Rodriguez-Iturbe et al., 1999:

$T(s)= \begin{cases}0 & \text { if } s \leq s_{w} \\ E_{\max } \frac{s-s_{w}}{s^{*}-s_{w}} & \text { if } s_{w}<s \leq s^{*} \\ E_{\max } & \text { if } s \geq s^{*}\end{cases}$

Leakage losses from the bottom of the soil layers are represented as in Eq. (7). Water percolating from the top layer, $L\left(s_{u}\right)$ enter the bottom layer. This phenomenon is different from the infiltration because it happens on slower time scales, due to gravity and not to root channeling.

In our approach, vegetation is modeled in Eq. (4) by an implicit-space logistic equation for the fraction of space, $b$, occupied by plants (Tilman, 1994). The pro-capite colonization rate, $g$, represents the ability of plants to colonize bare sites, and it combines the plant capacity to produce seeds and the germination probability of the seeds. The pro-capite colonization rate multiplies the fraction of space covered by vegetation, $b$, and the fraction of empty space, $1-b$, to express the total growth rate of the fraction of vegetated sites. The last term in Eq. (4) represents the fact that sites become empty due to the local extinction rate, $\mu$.

As in BA, the colonization rate depends on soil moisture. In principle, the colonization rate depends on the soil 

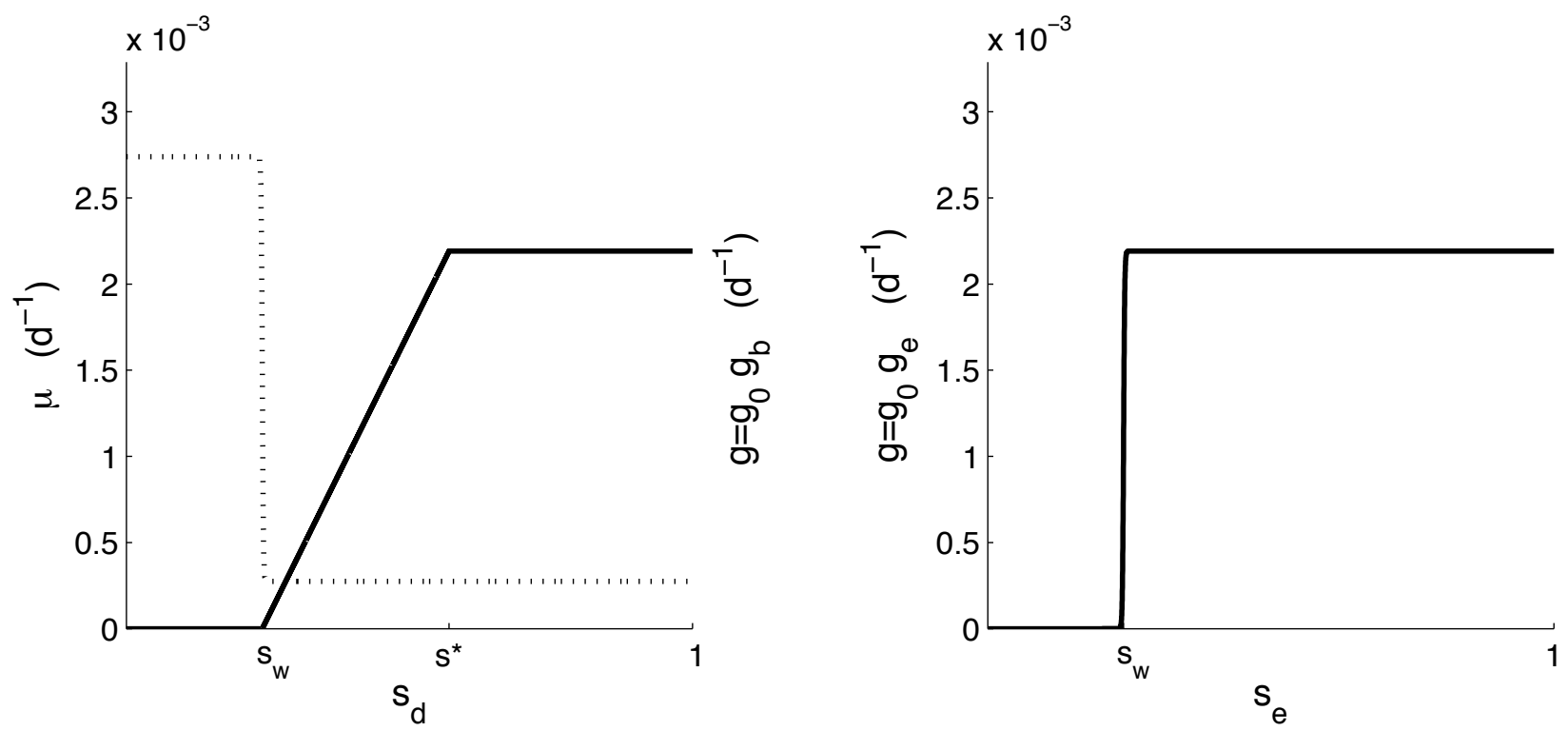

Fig. 1. Left panel: Continuous line, colonization rate depending on soil moisture in the root layer, $s_{d}, g=g_{0} g_{b}\left(s_{d}\right)$. Dotted line, mortality rate as a function of $s_{d}$. Right panel: Colonization rate depending on soil moisture of bare sites, $g=g_{0} g_{e}\left(s_{e}\right)$.

moisture of both empty and vegetated soil, and it is factorized in a seed production term, $g_{b}$, and a seed germination term, $g_{e}$ :

$g=g_{0} g_{e}\left(s_{e}\right) g_{b}\left(s_{b}\right)$.

Here, $g_{0}$ is the maximum colonization rate, $g_{e}\left(s_{e}\right)$ represents the seed establishment probability as a function of surface humidity in bare soil, and $g_{b}\left(s_{b}\right)$ represents the plant ability to produce seeds as a function of soil moisture in vegetated sites.

The seed production term, $g_{b}$, is assumed to vary with the soil moisture of the vegetated part, $s_{b}$. For simplicity, in the following we choose $s_{b}=s_{d}$. The results do not show qualitative changes when $s_{b}$ is chosen differently, e.g. equal to the weighted average of the moisture of the top and bottom soil layers in vegetated soil, $s_{b}=\left(s_{u} Z_{u}+s_{d} Z_{d}\right) /\left(Z_{u}+Z_{d}\right)$. The factor $g_{b}$ is supposed to be zero below a threshold corresponding to the wilting point, $s_{w}$, to grow linearly with soil moisture below the threshold for fully open stomata, $s^{*}$, and to become constant and equal to one for $s_{d}>s^{*}$ :

$g_{b}= \begin{cases}0 & \text { if } s_{d}<s_{w} \\ \frac{s_{d}-s_{w}}{s^{*}-s_{w}} & \text { if } s_{w} \leq s_{d}<s^{*} \\ 1 & \text { if } s^{*} \leq s_{d} \leq 1\end{cases}$

The factor $g_{e}$ represents the fact that seeds can germinate only when the humidity of the bare soil where they lie is sufficiently large. Dependence of seed germination on moisture of bare soil is modeled by assuming that $g_{e}$ varies from 0 to 1 with a hyperbolic tangent shape centered around a threshold value, chosen as the plant wilting point, $s_{w}$, analogously to the approach of BA:

$g_{e}\left(s_{e}\right)=\frac{1}{2}\left[1+\tanh \frac{s_{e}-s_{w}}{a}\right]$.

The mortality rate is assumed to depend on soil moisture on vegetated sites. In particular, $\mu$ depends on the bottom soil moisture $s_{d}$ : $\mu$ tends to the value $\mu_{1}$ for $s<s_{w}$ and to the value $\mu_{2}$ for $s>s_{w}$, where $\mu_{1}>\mu_{2}$. Following BA, we assume a hyperbolic tangent shape centered around $s_{w}$,

$\mu\left(s_{d}\right)=\frac{\mu_{1}+\mu_{2}}{2}+\frac{\mu_{1}-\mu_{2}}{2} \tanh \frac{s_{w}-s_{d}}{a}$.

For illustration, Fig. 1 shows $g_{0} g_{b}$ and $\mu$ as a function of $s_{d}$ and $g_{0} g_{e}$ as a function of $s_{e}$.

The values of the model parameters used in the following are reported in Tab. 1, and we have assumed to deal with annual vegetation on a loamy soil. Other choices of soil properties lead to qualitatively analogous results.

\section{Results}

In this section we report the results of our numerical exploration, focussing on the effect of vegetation feedbacks (infiltration and shading), and comparing the response of vegetation with different colonization strategies. We also estimate the impact of different rainfall regimes: either a constant input or temporally intermittent rainfall forcing. As in Laio et al. (2001) and in BA, precipitation intermittency is obtained by assuming independent, instantaneous rainfall 

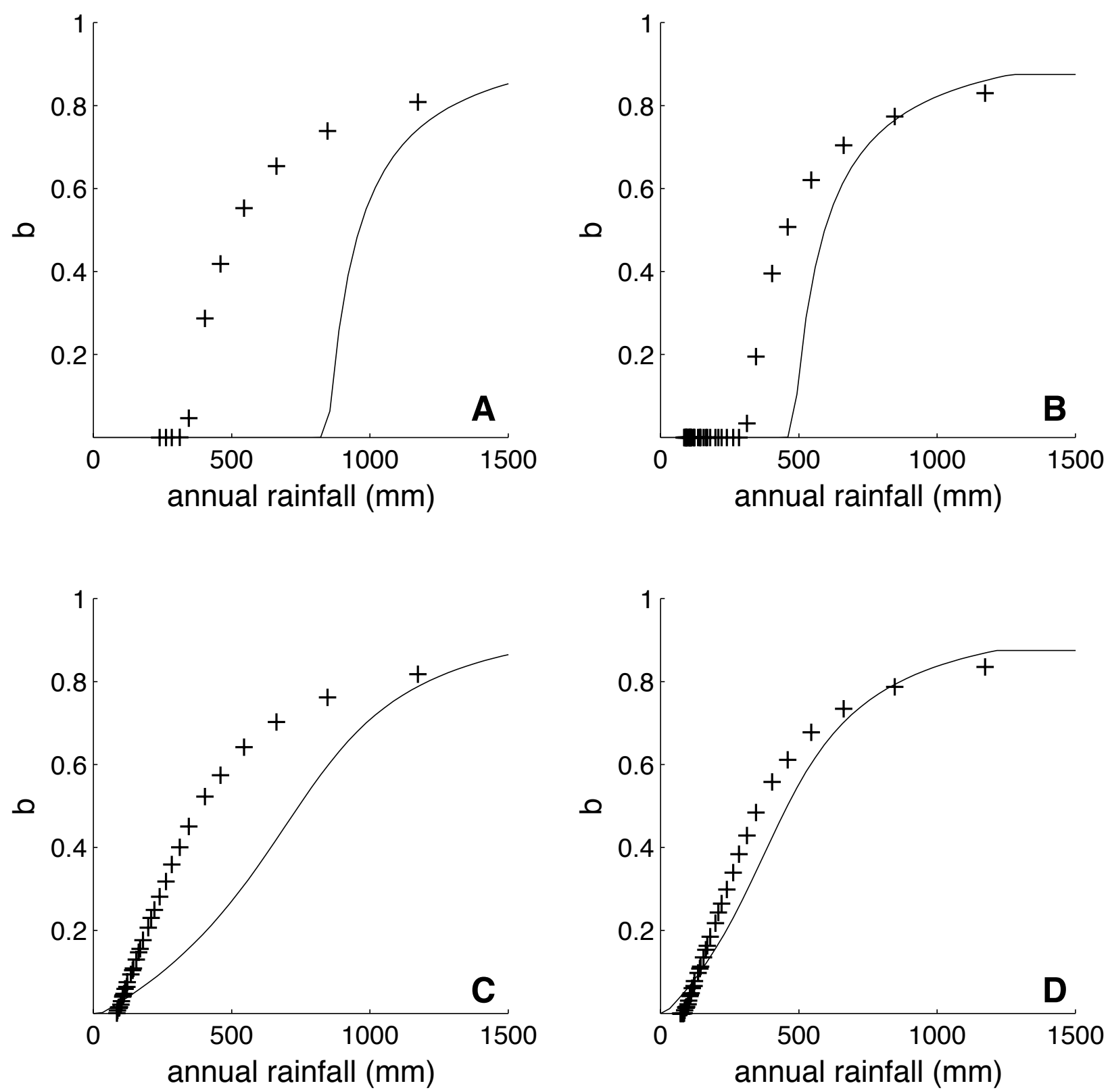

Fig. 2. Vegetation cover, $b$, versus annual rainfall, for the case where the colonization rate depends only on the soil moisture of the root layer $\left(s_{d}\right)$. Continuous lines represent equilibrium points in the case of constant rainfall, crosses represent average vegetation cover in the case of intermittent rainfall. The four panels refer to the following situations: (a) No vegetation feedbacks; (b) Presence of shading feedback only; (c) Presence of infiltration feedback only; (d) Presence of both infiltration and shading feedbacks. Rainfall is expressed by its annual value, i.e. the total rainfall during the wet season. The total annual rainfall is varied by changing the average interarrival time. Parameter values are reported in Table 1.

events distributed as a Poisson process with interarrival time $N$. The intensity of each event is extracted from an exponential distribution with average rainfall depth $r_{0}$. Rainfall occurs only during the wet season (200 days), and the soilvegetation system is assumed to be "frozen" during the dry season, see the discussion in BA.
3.1 Colonization rate dependent only on soil moisture in the root layer.

First we consider a case where the colonization rate, $g$, depends only on the vegetation ability to produce propagules, and not on the ability of seeds to germinate in bare soil. 

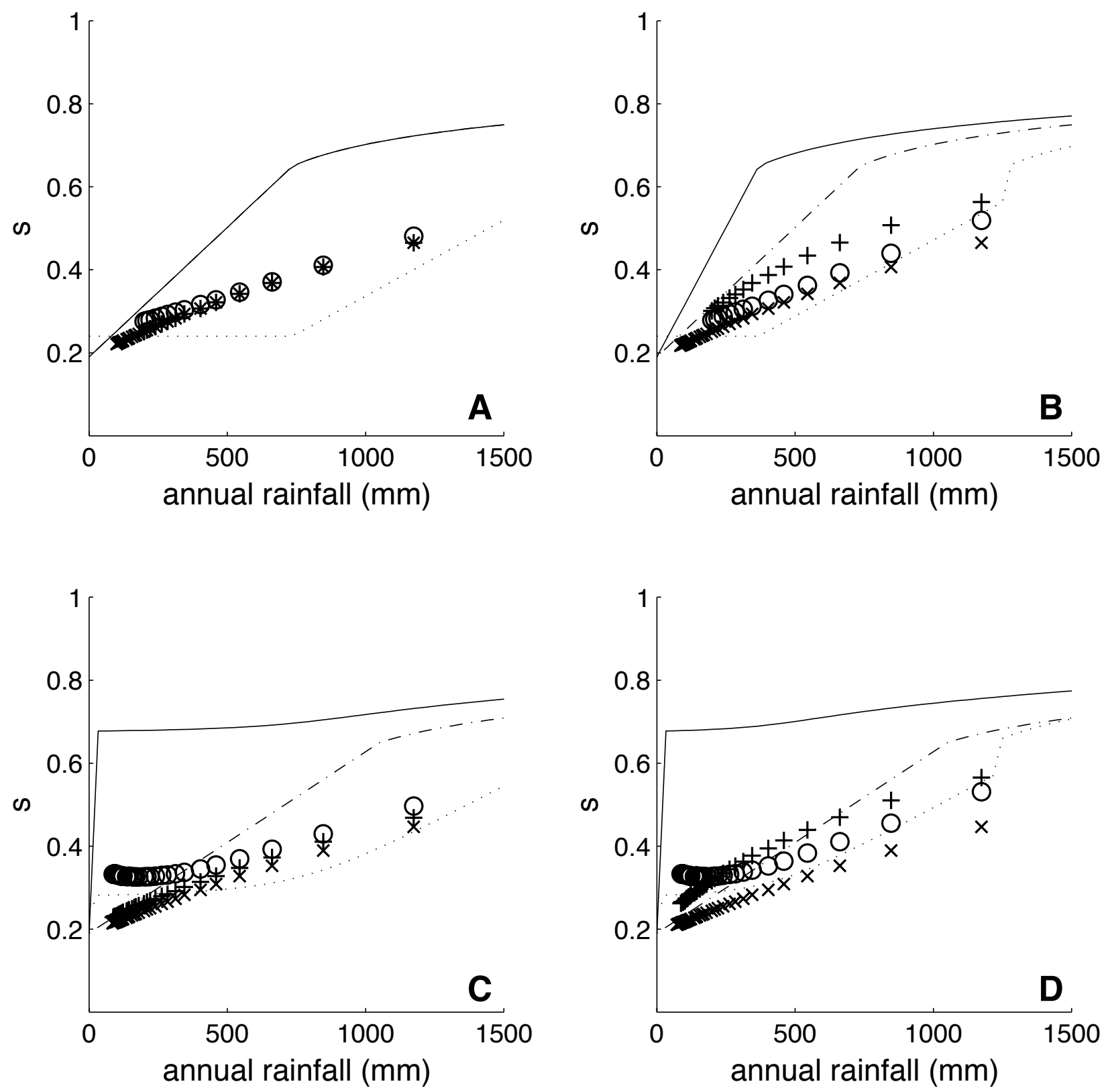

Fig. 3. Soil moisture, $s$, of bare and vegetated soil versus annual rainfall, for the case where the colonization rate depends only on the soil moisture of the root layer $\left(s_{d}\right)$. Lines represent equilibrium points in the case of constant rainfall (Continuous line, $s_{u}$, dotted line, $s_{d}$, dash-dotted line, $s_{e}$ ). Symbols represent the average soil moisture for intermittent rainfall: + marks for $s_{u}$, circles for $s_{d}$, X-signs for $s_{e}$. The values of $s_{u}$ and $s_{d}$ are not plotted when $b \rightarrow 0$. The four panels refer to the following situations: (a) No vegetation feedbacks; (b) Presence of shading feedback only; (c) Presence of infiltration feedback only; (d) Presence of both infiltration and shading feedbacks. Rainfall is expressed by its annual value, i.e. the total rainfall during the wet season. The total annual rainfall is varied by changing the average interarrival time. For parameter values see Table 1.

Consistently, we assume

$g\left(s_{b}\right)=g_{0} g_{b}\left(s_{b}\right)$,

with $g_{b}$ defined as in Eq. (12) and $g_{e}\left(s_{e}\right) \equiv 1$.
Figure 2a shows the vegetation behavior and Fig. 3a shows the soil moisture content when no vegetation feedbacks are introduced in the system: The bare soil is not crusted $(f=1)$ and vegetation does not decrease evaporation $(\sigma=1)$. In case of constant rainfall, vegetation is not present below about 
Table 1. List of model parameters and of their values. Units are expressed in millimeters (mm) and days (d). The chosen values are appropriate for annual vegetation (grasses) on loamy soil.

\begin{tabular}{clll}
\hline Symbol & \multicolumn{1}{c}{ Meaning } & Value & Units \\
\hline$n$ & Soil porosity & 0.45 & \\
$Z_{u}$ & Depth of the upper soil layer & 50 & $\mathrm{~mm}$ \\
$Z_{d}$ & Depth of the deeper soil layer & 250 & $\mathrm{~mm}$ \\
$s_{h}$ & Soil hygroscopic point & 0.19 & \\
$s_{w}$ & Soil wilting point & 0.24 & \\
$s^{*}$ & Soil moisture value at which plants start closing their stomata & 0.57 & \\
$s_{f c}$ & Soil field capacity & 0.65 & \\
$E_{m a x}$ & Maximum transpiration at $s^{*}$ & 4.5 & $\mathrm{~mm} \mathrm{~d}^{-1}$ \\
$E_{w}$ & Evaporation at wilting point & 0.4 & $\mathrm{~mm} \mathrm{~d}^{-1}$ \\
$E_{f c}$ & Evaporation at field capacity & 3.7 & $\mathrm{~mm} \mathrm{~d}^{-1}$ \\
$K_{S}$ & Saturated hydraulic conductivity & 200 & $\mathrm{~mm} \mathrm{~d}^{-1}$ \\
$r_{0}$ & Average rainfall depth & 15 & $\mathrm{~mm} \mathrm{~d}^{-1}$ \\
$\beta$ & Water retention parameter & 14.8 & \\
$g_{0}$ & Vegetation maximum colonization rate & $2.2 \times 10^{-3}$ & $\mathrm{~d}^{-1}$ \\
$\mu_{2}$ & Vegetation maximum extinction rate & $0.3 \times 10^{-3}$ & $\mathrm{~d}^{-1}$ \\
$\mu_{1}$ & Vegetation minimum extinction rate & $2.7 \times 10^{-3}$ & $\mathrm{~d}^{-1}$ \\
$a$ & Hyperbolic tangent width & 0.002 & \\
$f$ & Fraction of water infiltrating over bare soil & 0.7 & \\
$\sigma$ & Shading effect due to vegetation & 0.5 & \\
\hline
\end{tabular}

$800 \mathrm{~mm}$ per year. As in the case considered by BA, rainfall intermittency favors vegetation, shifting the threshold for vegetation persistence to about $400 \mathrm{~mm}$ per year. The surface soil moisture $s_{e}$ and $s_{u}$ are identical because no feedback is present in this case. In the intermittent case, surface soil moisture is lower than in the constant case, due to increased leakage and deep infiltration, which increase soil moisture in the root layer, $s_{d}$.

Figures $2 \mathrm{~b}$ and $3 \mathrm{~b}$ show the results when only a shading feedback is introduced, with $\sigma=0.5$. The equilibrium solution for constant rainfall is significantly affected by the shading feedback, with vegetation appearing for less than $500 \mathrm{~mm}$ per year. The moisture content of bare soil is not affected, but the soil moisture in the top vegetated layer, $s_{u}$, and, consequently, $s_{d}$, increase (Fig. $3 b$ ). Vegetation is again favored by intermittent rainfall but the advantage is lower than for the case with no feedbacks. Soil moisture in the bottom soil layer is higher under intermittent than under constant rainfall, but the difference is smaller than in the case without shading feedback.

Figures $2 \mathrm{c}$ and $3 \mathrm{c}$ show the results for the case where only the infiltration feedback is present: here, the biogenic crust is assumed to limit infiltration in bare soil to $70 \%$ of total precipitation $(f=0.7)$. Direct estimates in the Negev desert indicate that only about $60-80 \%$ of the precipitated water infiltrates over bare soil (M. Shachak, personal communication). Run-off water from crusted, bare soil infiltrates in vegetated patches (here, we assume that no run-off water is lost due to evaporation). This mechanism leads to vegetation presence at very low rainfall, both in the intermittent and in the constant precipitation cases, thanks to an increase in water availability in the vegetated parts (higher $s_{u}$ and $s_{d}$ ). This increase is accompanied by a decreased value of $s_{e}$, see Fig. 3c.

When the two feedbacks are considered together, as shown in Figs. 2d and 3d, the combined effect is a definite advantage for vegetation. Surprisingly, this advantage is more pronounced in the case of constant rainfall than for intermittent precipitation. In the constant case, vegetation is present even at values of total annual rainfall lower than $100 \mathrm{~mm}$ per year. The soil moisture in the root layer, $s_{d}$, is only slightly larger for intermittent rainfall than for constant rainfall. Under these conditions, rainfall intermittency plays a minor role: apparently, the presence of vegetation feedbacks reduces the role of rainfall intermittency.

3.2 Colonization rate dependent on soil moisture in the root layer and in bare soil.

A different approach consists in modeling vegetation colonization rate as dependent on both seed establishment probability and plant status. Seeds germinate over bare soil, and in this version of the model we assume that the colonization is expressed as

$g=g_{0} g_{e}\left(s_{e}\right) g_{b}\left(s_{b}\right)$

with $g_{e}$ as in (13) and $g_{b}$ as in (12).

The case without feedbacks, shown in Figs. $4 \mathrm{a}$ and $5 \mathrm{a}$, is identical to the case without dependence on bare soil moisture (Figs. 2a-3a), because bare soil limits vegetation growth 

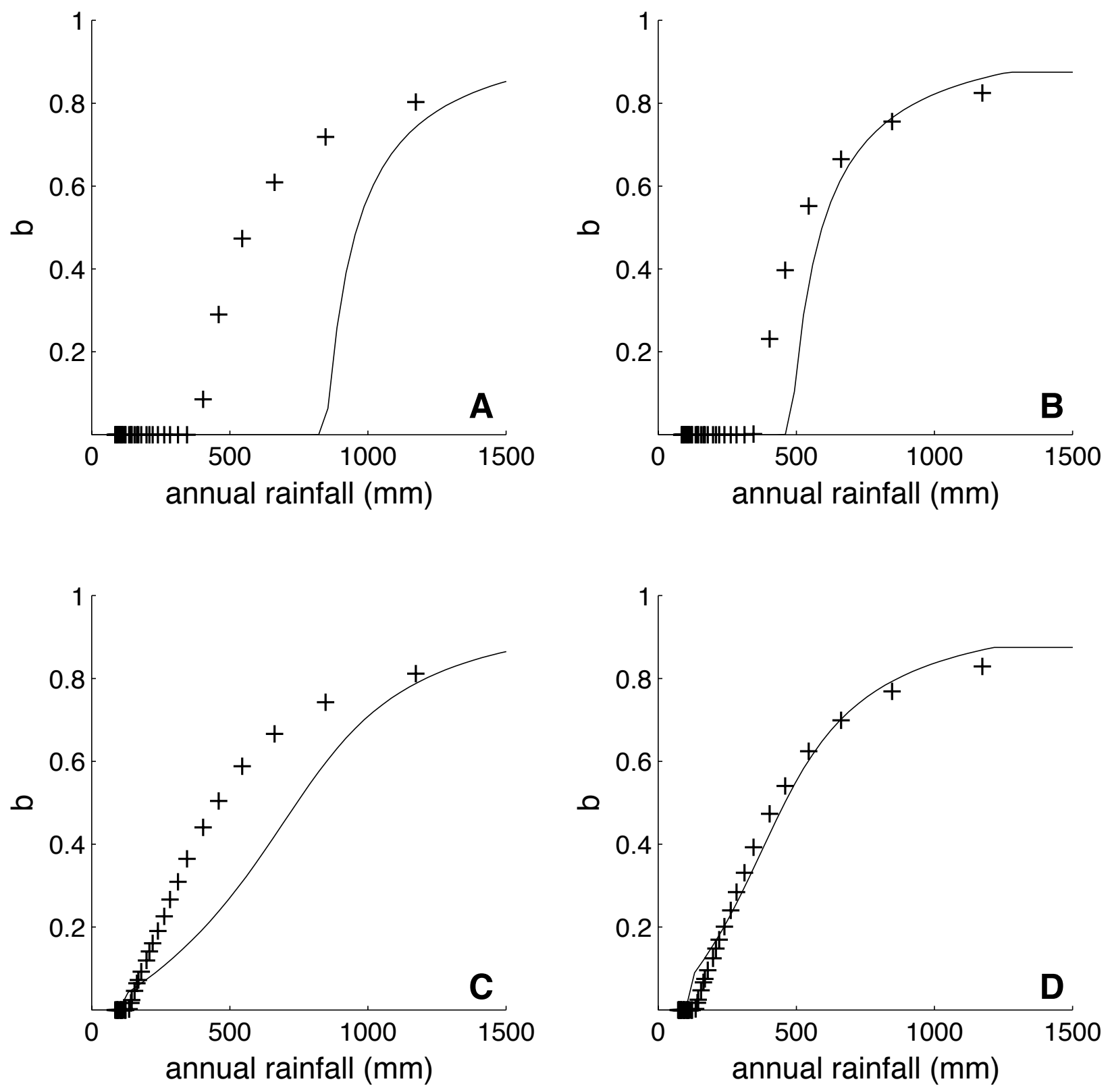

Fig. 4. Vegetation cover, $b$, versus annual rainfall, for the case where the colonization rate depends on both the soil moisture of the root layer in vegetated soil and on that of bare soil. Continuous lines represent equilibrium points in the case of constant rainfall, crosses represent the average vegetation cover in the case of intermittent rainfall. The four panels refer to the following situations: (a) No vegetation feedbacks; (b) Presence of shading feedback only; (c) Presence of infiltration feedback only; (d) Presence of both infiltration and shading feedbacks. Rainfall is expressed by its annual value, i.e. the rainfall during the wet season. The total annual rainfall is varied by changing the average interarrival time. Parameter values are reported in Table 1.

only at low water amounts, for $s_{e}<s_{w}$, where in any case $g_{b} \equiv 0$, owing to the fact that $s_{d} \leq s_{w}$, and thus vegetation cover is zero. Also in the cases with a presence of vegetation feedbacks the results are very similar to those obtained without dependence on bare soil humidity $\left(g=g_{0} g_{b}\right)$. In particular, Fig. 5a and b are identical to Fig. 3a and b. Indeed, soil moisture dynamics changes with vegetation cover only when the differential infiltration between vegetated and bare surface is included (Fig. 3c,d).

The effect of the dependence of the colonization rate on $s_{e}$ manifests itself in two features. First, under constant rainfall conditions, a limit is imposed to vegetation growth when $s_{e}$ is lower than $s_{w}$. As shown in Fig. $4 \mathrm{c}$ and d, $b>0$ only above a value of annual rainfall of about $100 \mathrm{~mm}$ per year. Second, 

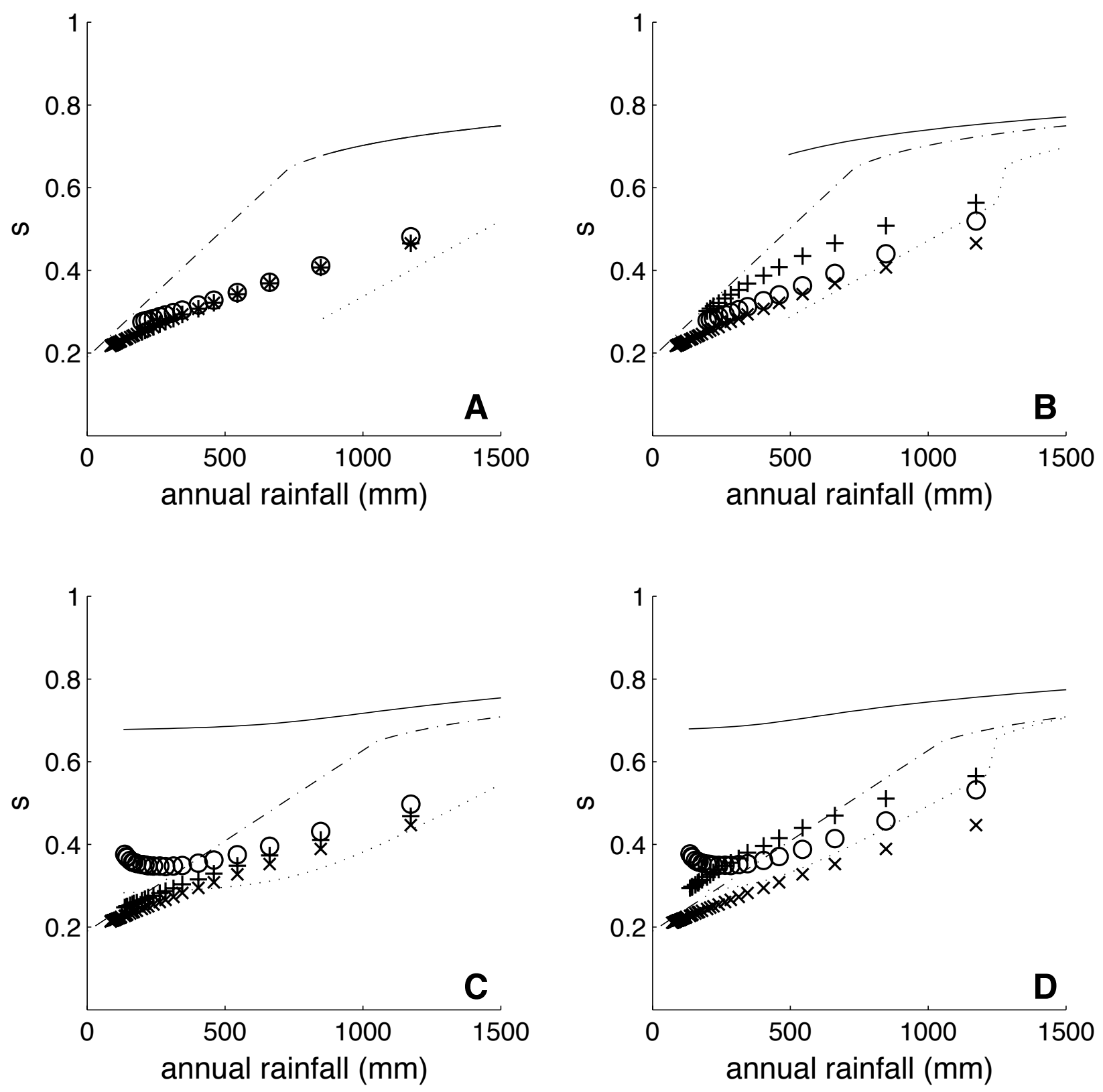

Fig. 5. Soil moisture, $s$, of bare and vegetated soil versus annual rainfall, for the case where the colonization rate depends on both the soil moisture of the root layer in vegetated soil and on that of bare soil. Lines represent equilibrium points in the case of constant rainfall (Continuous line, $s_{u}$, dotted line, $s_{d}$, dash-dotted line, $s_{e}$ ), symbols represents average soil moisture in the case of intermittent rainfall (+marks for $s_{u}$, circles for $s_{d}$, X-signs for $s_{e}$ ). The values of $s_{u}$ and $s_{d}$ are not plotted when $b \rightarrow 0$. The four panels refer to the following situations: (a) No vegetation feedbacks; (b) Presence of shading feedback only; (c) Presence of infiltration feedback only; (d) Presence of both infiltration and shading feedbacks. Rainfall is expressed by its annual value, i.e. the rainfall during the wet season. The total annual rainfall is varied by changing the average interarrival time. For parameter values see Table 1 .

the beneficial effect of rainfall intermittency on vegetation is smaller, and it almost disappears when both feedbacks are considered.

\section{Discussion}

The results illustrated in the previous section suggest a number of considerations on the role of vegetation feedbacks, on rainfall intermittency and on their interplay.

When $g$ depends only on $s_{d}$, the minimum constant rainfall 
needed to guarantee vegetation survival is $\approx 800 \mathrm{~mm}$ per year, corresponding to the threshold at which $s_{d}$ grows above $s_{w}$, and thus $g>0$. In the absence of any vegetation feedbacks, the non-linear dependence of $g$ on $s_{d}$ leads to a significant beneficial effect of rainfall intermittency, similarly to what has been discussed in detail by BA. The situation is analogous when $g$ depends on both $s_{e}$ and $s_{d}$.

In the presence of vegetation feedbacks, the situation can change. Vegetation limits surface evaporation, increasing water in the top layer of vegetated soil. Deep infiltration and leakage grow, thus increasing deep water storage and advantaging vegetation growth owing to the dependence of $g$ on $s_{d}$. Interestingly, Figs. $2 \mathrm{~b}$ and $4 \mathrm{~b}$ show that the larger advantage due to shading occurs when rainfall is homogeneously distributed in time.

The other effect of vegetation is to facilitate infiltration from rainfall, as compared to bare soil where crusts of either physical or biological origin limit infiltration (West, 1990; Rietkerk et al., 2002; Gilad et al., 2004). This effect is always favorable to vegetation because it increases biomass locally. On the other hand, it is more difficult for seeds to germinate over dryer soils. When $g$ depends only on $s_{d}$, the infiltration feedback has a strong positive effect on $b$, which remains positive, albeit small, even at very low annual rainfall. When $g$ depends also on $s_{e}$, for a constant precipitation input vegetation appears at slightly larger values of annual rainfall, but still much smaller than for the case without the infiltration feedback.

The infiltration feedback is considered to be one of the reasons for vegetation pattern formation in spatially explicit models, bringing advantages to vegetation (von Hardenberg et al., 2001; Rietkerk et al., 2004). In these models, biomass grows locally due to the larger amount of water available for root uptake. One can include this effect by considering higher colonization ability when the soil in the root layer is wetter, representing the fact that plants can colonize more easily sites around them. Introducing this dependency, infiltration and shading feedbacks have larger effects, in agreement with the results obtained with explicit-space models.

For the case with the two vegetation feedbacks acting together, the advantage due to the feedbacks is larger in case of constant rainfall than for intermittent rainfall. Both feedbacks increase surface moisture in the vegetated parts and therefore deep water storage. A similar effect is obtained in the case of intermittent rainfall occurring in high-amplitude intermittent pulses. Vegetation is favored by soil moisture increasing up to $s^{*}$ in the root layer, but it does not get any further advantage when soil moisture grows above $s^{*}$. The effects of either soil-vegetation feedbacks or of rainfall intermittency are already quite large when considered individually, and adding them just pushes soil moisture above the level where vegetation is not favored much further. Rainfall intermittency can even be disadvantageous for vegetation, if compared to constant rainfall. When feedbacks are very effective, such as highly limited infiltration over bare soil or strong vegetation shading effect (e.g., $f=0.3$ or $\sigma=0.2$ ), the combined effect of feedbacks and intermittency saturates the vegetated soil, and the larger water losses damage vegetation.

In general, we observe that the feedbacks are more advantageous, in both rainfall regimes, when $g$ does not depend on bare soil moisture, as this latter is significantly lowered by the infiltration feedback. This effect is easily understood if we consider a case where the colonization rate, $g$, depends only on soil moisture in bare soil, i.e.:

$g\left(s_{e}\right)=g_{0} g_{e}\left(s_{e}\right)$

where $g_{e}\left(s_{e}\right)$ varies as in Eq. (13), with a threshold around $s_{e}=s_{w}$. Such a choice would correspond to a case where the existence of a seed bank is a plausible hypothesis: Seeds are present everywhere and they are ready to germinate under favorable conditions (e.g. Oren, 2001). Under such conditions, dependence of colonization ability on just the soil moisture in empty sites is a possible modeling choice, and moisture in the root layer comes into play only in plant mortality.

Model runs with this choice for $g$ indicate that in this case the infiltration and shading feedbacks favor vegetation only partially, diminishing mortality, while vegetation spread to new sites is limited because of the increased difficulty in colonizing bare soil. The advantage brought to vegetation by feedbacks is therefore rather minor, especially for intermittent rainfall. In fact, in this case the infiltration feedback could even reduce the spatial spread of vegetation, favoring instead the growth of new vegetation in patches already occupied by older plants. When $g$ depends on soil moisture in both vegetated and bare soil, on the other hand, the disadvantage brought in by the lower bare soil moisture when the infiltration feedback is present is largely compensated by the correspondent advantages due to larger soil moisture in vegetated soil. In this case, one does not observe large differences with respect to the case where the colonization rate depends only on soil moisture in vegetated patches.

The results discussed in this work indicate that the influence of vegetation feedbacks is larger when rainfall is kept constant in time. Many studies dealing with the importance of vegetation feedbacks, both with implicit and explicit spatial structure, considered rainfall as a constant input (e.g. Zeng et al., 2006; von Hardenberg et al., 2001; Rietkerk et al., 2002). Here the importance of rainfall intermittency is underlined again, showing that part of the effects due to the feedbacks (namely, deep water storage) can be obtained, without feedbacks, when rainfall occurs in intermittent pulses. Depending on the vegetation strategy and on the type of vegetation feedbacks present in the system, rainfall intermittency can thus be more or less beneficial to vegetation.

\section{Summary and conclusion}

In this work we have discussed a simple vegetation-soil moisture model for drylands. The model includes two effects 
which were discarded in most previous models: Evaporation occurs from the top layer only, allowing water storage in the deeper root layer, and vegetation feedbacks lead to reduced evaporation from vegetated areas and to differential infiltration between vegetated and empty sites.

Plant colonization behavior has been assumed to follow different strategies, depending on soil moisture in bare soil, to represent seed germination abilities, and on moisture in vegetated soil, to represent seed production. Depending on the functional form of the colonization rate, the effect of infiltration or shading feedbacks is different. In general, however, we find that in this model the beneficial effects of rainfall intermittency and of vegetation feedbacks are somehow mutually exclusive: vegetation feedbacks act at their maximum strength for a constant rainfall input, and rainfall intermittency loses most of its beneficial power when strong vegetation feedbacks are present. One can thus expect that plants living in areas with different rainfall climatologies (more or less intermittent for the same value of rainfall volume) may have evolved different strategies.

Acknowledgements. We are grateful to F. D'Andrea, S. Dekker, J. von Hardenberg, A. Porporato, M. Rietkerk and M. Shachak for useful discussions and comments on this work.

Edited by: S. Manfreda

\section{References}

Allen, R. G., Pereira, L. S., Smith, M., Raes, D., and Wright, J. L.: FAO-56 Dual Crop Coefficient Method for Estimating Evaporation from Soil and Application Extensions, J. Irrig. Drain. E., 131, 2-13, 2005.

Baudena, M., Boni, G., Ferraris, L., von Hardenberg, J., and Provenzale, A.: Vegetation response to rainfall intermittency in drylands: Results from a simple ecohydrological box model, Adv. Water Res., 30, 1320-1328, 2007.

Borgogno, F., D’Odorico, P., Laio, F., and Ridolfi, L.: Effect of rainfall interannual variability on the stability and resilience of dryland plant ecosystems, Water Resour. Res., 43, W06411, doi: 10.1029/2006WR005314, 2007.

D’Odorico, P., Laio, F., and Ridolfi, L.: Noise-induced stability in dryland plant ecosystems, P. Natl. A. Sci., 102, 10819-10822, 2005.

Gilad, E., von Hardenberg, J., Provenzale, A., Shachak, M., and Meron, E.: Ecosystem Engineers, in : from Pattern Formation to Habitat Creation, Phys. Rev. Lett., 93, 098105, 2004.

Gilad, E., von Hardenberg, J., Provenzale, A., Shachak, M., and Meron, E.: A mathematical model of plants as ecosystem engineers, J. Theor. Biol., 244, 680-691, 2007.
Laio, F., Porporato, A., Ridolfi, L., and Rodriguez-Iturbe, I.: Plants in water controlled ecosystems, in: active role in hydrological processes and response to water stress II. Probabilistic soil moisture dynamics, Adv. Water Res., 24, 707-723, 2001.

Levins, R.: Some demographic and genetic consequences of environmental heterogeneity for biological control, Bulletin of the Entomological Society of America, 15, 237-240, 1969.

Mahrt, L. and Pan, H.: A two layer model of soil hydrology, Bound.-Lay. Meterorol., 29, 1-20, 1984.

Oren, Y.: Patchiness, disturbances, and the flows of matter and organisms in an arid landscape: a multiscale experimental approach, $\mathrm{PhD}$ thesis, Ben-Gurion University of the Negev, BeerSheva, Israel, 2001.

Rietkerk, M. and van de Koppel, J.: Alternate stable states and threshold effects in semi-arid grazing systems, Oikos, 79, 69-76, 1997.

Rietkerk, M., Boerlijst, M. C., van Langevelde, F., HilleRisLambers, R., van de Koppel, J., Kumar, L., Prins, H. H. T., and Roos, A. M. D.: Self-Organization of Vegetation in Arid Ecosystems, American Naturalist, 160, 524-530, 2002.

Rietkerk, M., Dekker, S. C., de Ruiter, P. C., and van de Koppel, J.: Self-Organized Patchiness and Catastrophic Shifts in Ecosystems, Science, 305, 1926-1929, 2004.

Rigby, J. and Porporato, A.: Simplified stochastic soil moisture models: a look at infiltration, Hydrol. Earth Syst. Sci., 10, 861871,2006 , http://www.hydrol-earth-syst-sci.net/10/861/2006/.

Rodriguez-Iturbe, I. and Porporato, A.: Ecohydrology of water controlled ecosystems: soil moisture and plant dynamics, Cambridge University Press, doi:10.2277/0521819431, 2005.

Rodriguez-Iturbe, I., D’Odorico, P., Porporato, A., and Ridolfi, L.: On the spatial and temporal links between vegetation, climate and soil moisture, Water Resour. Res., 35, 3709-3722, 1999.

Scanlon, T. M. and Albertson, J. D.: Inferred controls on tree/grass composition in a savanna ecosystem, in: Combining 16-year normalized difference vegetation index data with a dynamic soil moisture model, Water Resour. Res., 39, 1224, doi:10.1029/ 2002WR001881, 2003.

Tilman, D.: Competition and biodiversity in spatially-structured habitats, Ecology, 75, 2-16, 1994.

von Hardenberg, J., Meron, E., Shachak, M., and Zarmi, Y.: Diversity of Vegetation Patterns and Desertification., Phys. Rev. Lett., 87, 198 101, 2001.

Walker, B. H., Ludwig, D., Holling, C. S., and Peterman, R. M.: Stability of semi-arid savanna grazing system, J. Ecol., 69, 473498, 1981.

West, N. E.: Structure and function of soil microphytic crusts in wildland ecosystems of arid to semi-arid regions, Adv. Ecol. Res., 20, 179-223, 1990.

Zeng, X., Wang, A., Zeng, Q., Dickinson, R. E., Zeng, X., and Shen, S. S. P.: Intermediately complex models for the hydrological interactions in the atmosphere-vegetation-soil system, Adv. Atmos. Sci., 23, 127-140, 2006. 